SCIEntiæ studia, São Paulo, v. 11, n. 1, p. 119-39, 2013

\title{
st \\ Refinamentos da interpretação da complementaridade \\ a partir do experimento de Afshar
}

Osvaldo Pessoa Júnior

\begin{abstract}
电
RESUMO

O experimento de Afshar foi proposto recentemente como sendo uma violação do princípio de complementaridade. Reconhecendo a novidade trazida pelo experimento, argumentamos que ele permite um refinamento de tal princípio, a partir do estabelecimento de dois pontos: (1) a possibilidade de modificar o "tipo" de fenômeno (onda ou partícula) sem alterar o estado quântico, e (2) a constatação de que o tipo de fenômeno, associado a um quantum detectado, refere-se a um trecho determinado percorrido pelo objeto quântico. O primeiro ponto é explorado em interferômetros de Mach-Zehnder, com dispositivos de polarização e na montagem dupla de Unruh. O segundo ponto salienta que um fenômeno pode ser corpuscular com respeito a uma região e ondulatório com respeito a outra, estando a novidade de Afshar na proposta de uma maneira de constatar ambos simultaneamente. A restrição (1) acaba tendo um efeito desprezível no experimento de Afshar.
\end{abstract}

PaLAVras-chave • Filosofia da física. Fundamentos da mecânica quântica. Complementaridade. Dualidade onda-partícula. Estado quântico. Interferômetro de Mach-Zehnder. Retrodição. Experimento de Afshar. Experimento de Unruh.
Dado que o elétron interage continuamente com muitas espécies diferentes de sistemas, cada qual desenvolvendo potencialidades dife- rentes, o elétron será submetido a transforma- ções contínuas entre suas diferentes formas possíveis de comportamento (isto é, onda ou partícula) (Bohm, 1951, p. 139).

\section{INTRODUÇÃO}

Nos últimos anos, tem havido uma discussão a respeito do experimento de Afshar, no qual o princípio de complementaridade de Bohr teria sido aparentemente violado, em um arranjo experimental que manifestaria simultaneamente propriedades nitidamente 
ondulatórias e nitidamente corpusculares. Vários autores têm contestado a afirmação do físico iraniano Shahriar Afshar, que realizou experimentos confirmando suas próprias previsões na Universidade de Harvard em 2003, onde trabalhava como pesquisador visitante (cf. Afhsar, 2003). No presente artigo, examina-se esse experimento à luz de duas constatações: (1) é possível modificar o "tipo" de fenômeno (onda ou partícula) sem alterar o estado quântico (seções 5 e 6), e (2) o tipo de fenômeno, associado a um quantum detectado, refere-se a um trecho determinado percorrido pelo objeto quântico (seção 8). Com isso, conclui-se que o experimento de Afshar traz um importante refinamento para a interpretação da complementaridade de Niels Bohr.

\section{Complementaridade DE ARRANJos EXPERIMENTAis}

O princípio de complementaridade é central para a interpretação ortodoxa da mecânica quântica desenvolvida por Niels Bohr. Há, de fato, três tipos diferentes de "complementaridade" nos escritos de Bohr (cf. Pessoa, 2000a, p. 65-6, 2003, p. 93-5), mas a que iremos analisar aqui é a complementaridade entre arranjos experimentais (a dualidade onda-partícula). De acordo com essa tese, um experimento quântico pode ser representado ou em um quadro corpuscular ou em um quadro ondulatório, nunca em ambos ao mesmo tempo. Esses aspectos da experiência seriam mutuamente excludentes e, além disso, eles forneceriam uma descrição exaustiva do objeto atômico. Deixando de lado a questão da exaustividade, examinemos o que significa um fenômeno ser corpuscular ou ondulatório.

\section{O interferômetro de MAGh-Zehnder}

Um "fenômeno", de acordo com a definição de Bohr (1995 [1949], p. 51) envolve tanto o objeto quântico quanto o arranjo experimental, e só é completado quando ocorre uma medição (com registro macroscópico, que pode ser nulo, cf. Pessoa, 2003, p. 54). A necessidade de esperar a conclusão da medição foi ilustrada por Wheeler (1978), em sua proposta de um "experimento de escolha demorada", envolvendo o interferômetro de Mach-Zehnder, mostrado na figura 1.

Para entender como esse aparelho funciona, consideremos a mecânica ondulatória clássica. Um feixe de luz, que, por simplicidade, é representado por um trem de onda monocromático unidimensional, é dividido em dois componentes no espelho semirrefletor $S_{1}$. O componente transmitido segue pelo caminho $A$, é refletido no es- 


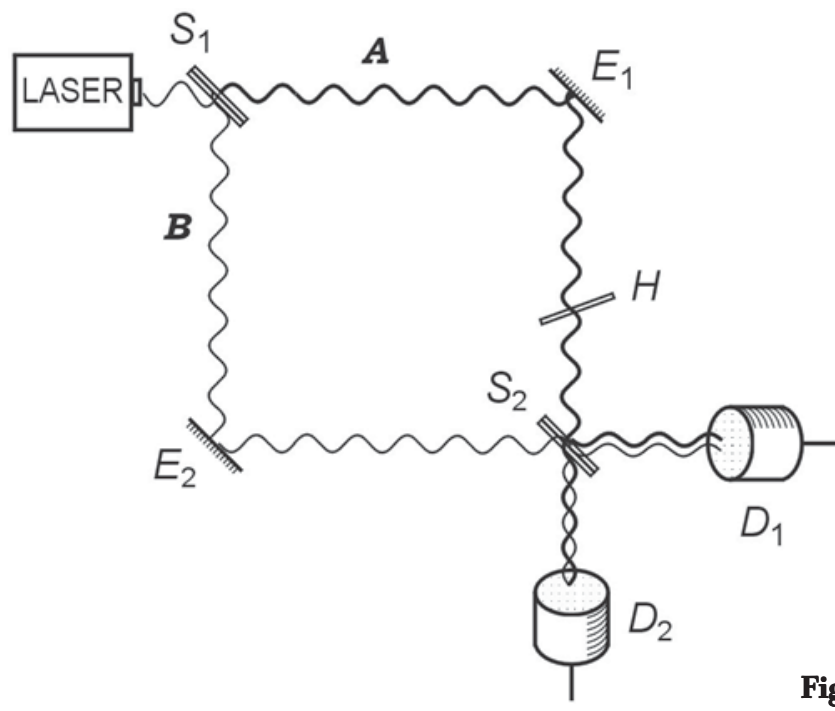

Figura 1. Interferômetro de Mach-Zehnder.

pelho $E_{1}$ e incide no espelho semirrefletor $S_{2}$. O componente que é refletido para o caminho $B$ viaja a mesma distância e é também dividido em $S_{2}$. Ajustando-se a lâmina de vidro $H$ em um ângulo adequado (regulando a fase do componente $A$ ), garante-se que as amplitudes que rumam para o detector $D_{2}$ interfiram destrutivamente, ao passo que aquelas que se dirigem para $D_{1}$ superponham construtivamente. Assim, toda luz incidente termina em $D_{1}$, e nada é detectado em $D_{2}$.

Tal experimento é adequadamente descrito pela mecânica ondulatória clássica. A teoria quântica torna-se necessária apenas quando a intensidade do feixe incidente é muito reduzida, e os detectores usuais são substituídos por detectores de alta sensibilidade, como fotomultiplicadores. Pode-se assim, na detecção, discernir quanta individuais de luz (fótons). As amplitudes de probabilidade para detecção no regime quântico são idênticas às amplitudes de onda eletromagnética do caso clássico. Assim, todos os fótons são detectados em $D_{1}$ e nenhum em $D_{2}$.

\section{TiPos de Fenômenos: ONDA OU PARTícula}

O experimento quântico descrito acima é um típico "fenômeno ondulatório". Uma maneira de justificar essa denominação é argumentando que as amplitudes finais de probabilidade só podem ser explicadas supondo-se os conceitos ondulatórios de interferência construtiva e destrutiva. Mas há também uma justificação operacional para 
essa expressão: ${ }^{\mathbf{1}}$ se o defasador $H$ da figura 1 for lentamente girado, de forma que a fase $f$ do componente $A$ varie lentamente com o tempo, as taxas de contagem (intensidades) em cada detector irão variar temporalmente de maneira proporcional ao $\cos ^{2} \phi$, constituindo um típico padrão de interferência (neste caso, temporal, não espacial como no experimento da dupla fenda) (cf. Grangier et al., 1986; Hellmuth et al., 1987).

Assim, o fenômeno é ondulatório. Não seria ele também corpuscular, já que os quanta individuais podem ser discernidos? A resposta tradicional é que não: ${ }^{2}$ para o fenômeno ser "corpuscular", deve ser possível associar uma trajetória bem definida ao quantum detectado (trajetória esta associada a um movimento uniforme, na ausência de campos externos). No experimento da figura 1, somos, em princípio, incapazes de inferir qual teria sido a trajetória de um fóton específico. Além disso, se supusermos que o fóton seguiu, por exemplo, pelo caminho $A$, e que nada seguiu pelo caminho $B$, então haveria uma probabilidade $1 / 2$ de detecção em $D_{2}$, o que é diferente do que acontece de fato no experimento.

Considere-se agora o que acontece se o espelho semirrefletor $S_{2}$ é removido. A interferência entre os feixes deixa de ocorrer, o componente $A$ cai em $D_{2}$, enquanto o feixe $B$ ruma para $D_{1}$, de forma que metade dos fótons incidentes é detectada em cada fotomultiplicadora (que supomos ter eficiência perfeita). Se o caminho $B$ for bloqueado, apenas $D_{2}$ irá disparar (e analogamente para $A$ ). Assim, para cada fóton detectado, pode-se inferir qual a trajetória seguida por ele, e é isso que caracteriza um "fenômeno corpuscular".

A complementaridade de arranjos experimentais, portanto, pode ser enunciada como a impossibilidade de se ter, ao mesmo tempo, nítidos efeitos de interferência e trajetórias sem ambiguidade. ${ }^{3}$ Trata-se de uma afirmação empiricamente refutável, de forma que o estudo da dualidade onda-partícula, por si só, não nos compromete com nenhuma interpretação específica da mecânica quântica.

John Wheeler (1978) chamou a atenção para o fato de que o cientista pode escolher se o fenômeno será ondulatório ou corpuscular (mantendo ou removendo $S_{2}$ ) mesmo depois que o pacote de onda (associado ao objeto quântico) tenha passado por $S_{1}$ e entrado no interferômetro. Esse experimento de "escolha demorada" mostra que o

\footnotetext{
1 Há uma dificuldade básica com essa estratégia operacional: a variação de fase também modifica o experimento. Deve-se, portanto, garantir que esse tipo de modificação "preserva o tipo de fenômeno". Para um estudo de outras modificações que preservam o tipo de fenômeno, cf. Pessoa, 2000a.

$2 \mathrm{O}$ fato de que deteç̧ões no regime quântico envolvem quanta individuais é, de acordo com Bohr (2000 [1928], p. 136), uma expressão do "postulado quântico" de Planck, e não da complementaridade. Veremos, na seção 8, como unificar os dois.

3 Os adjetivos "nítido" e "sem ambiguidade" foram introduzidos para que se excluam os fenômenos intermediários, que misturam aspectos ondulatórios e corpusculares, mas que também vêm em pares complementares (cf. Pessoa, 2003, p. 112-6).
} 
Refinamentos da interpretação da Gomplementaridade...

tipo de fenômeno quântico (onda ou partícula) só é estabelecido após a medição ser completada. Antes da deteç̧ão, nada pode ser dito com respeito à natureza do objeto quântico. Nesse sentido, a interpretação da complementaridade é anti-realista ou "fenomenalista" (a teoria quântica só descreveria o que é observável).

Voltemos ao fenômeno corpuscular obtido através da remoção de $S_{2}$ do interferômetro de Mach-Zehnder (ver fig. 1). É realmente óbvio que um fóton detectado em $D_{1}$ seguiu a trajetória do caminho $B$ ? Se o leitor considera que sim, então ele está implicitamente aceitando o que se chama de retrodição, uma inferência para o passado (neste caso, para trajetórias passadas). Tanto Bohr quanto Heisenberg estavam cientes de que a retrodição é uma tese interpretativa (não factual), que não leva a nenhuma contradição com a experiência, mas que é de "um caráter puramente especulativo" ( Heisenberg, 1930, p. 20; cf. Bohr, 2000 [1928], p. 145). Salientaram isso porque a retrodição leva à conclusão de que o princípio de incerteza não se aplica ao passado (em certos fenômenos corpusculares, cf. Pessoa, 2003, p. 81). Mas mesmo assim, a partir de 1935, a retrodição foi implicitamente incorporada à interpretação da complementaridade de Bohr, em sua definição de fenômeno corpuscular.

\section{O INTERFERÔMETRO GOM POLARIZADORES}

Façamos agora uma modificação no arranjo experimental visto acima, introduzindo polarizadores mutuamente ortogonais nos braços do interferômetro de Mach-Zehnder. Para começar, vamos supor que o feixe que sai do laser está linearmente polarizado no ângulo de $\circ^{\circ}$. Ao invés de usar polarizadores, que absorvem parte do feixe, introduzimos no caminho $B$ um rotor de polarização $R$ que gira o plano de polarização em $\pi / 2$

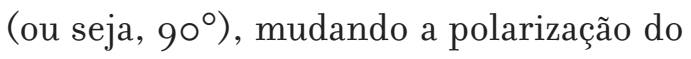
componente desse braço de $0^{\circ}$ a $90^{\circ}$ (ver fig. 2). Além disso, cada detector da figura 1 é substituído por um analisador de polarização, ou seja, um prisma birrefringente que separa qualquer feixe incidente em componentes polarizadas a $0^{\circ}$ e a $90^{\circ}$, cada qual incidindo em um detector separado.

Figura 2. Interferômetro de Mach-Zehnder com dispositivos polarizadores. A inserção do filtro polarizador $F$ não altera o estado quântico e não absorve luz, mas muda o tipo de fenômeno associado aos fótons detectados em $D_{1} \mathbf{e} D_{2}$.

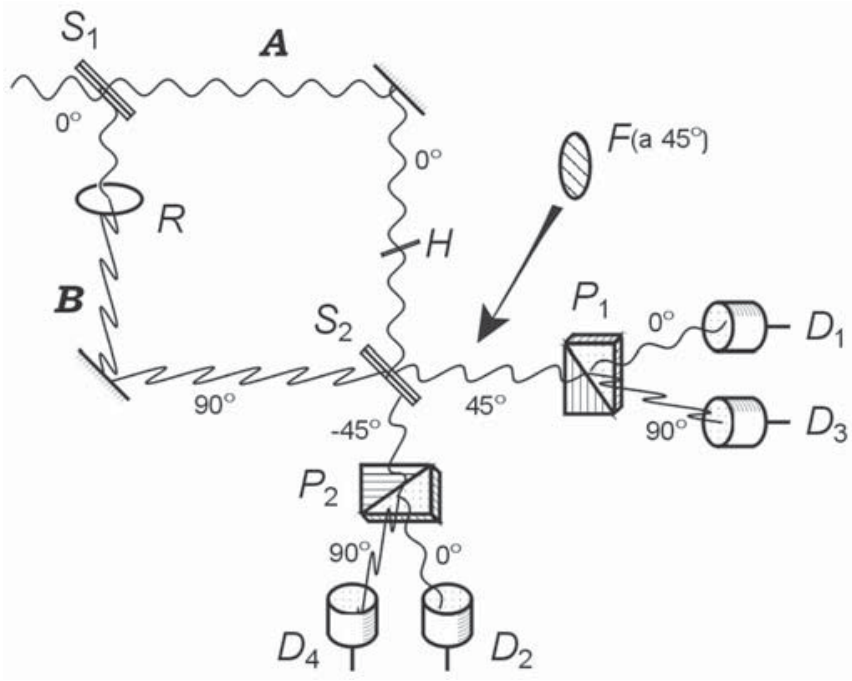


No regime de intensidades moderadas e detectores ordinários, a mecânica ondulatória clássica representa o componente em $A$ como uma onda eletromagnética oscilando transversalmente na direção específica $x\left(0^{\circ}\right)$, ao passo que o componente em $B$ é uma onda transversal oscilando ao longo da direção ortogonal $\boldsymbol{y}\left(90^{\circ}\right)$. Após a recombinação no espelho semirrefletor $S_{2}$, os componentes ortogonais não interferem construtiva ou destrutivamente, como no arranjo anterior da figura 1. Haverá superposição vetorial das ondas que oscilam nos eixos $\boldsymbol{x}$ e $\boldsymbol{y}$, e a onda resultante estará linearmnete polarizada a $45^{\circ}$ (no caso dos componentes rumando para o prisma $P_{1}$ ) ou a $-45^{\circ}$ (para o componente que vai para o analisador $P_{2}$ ). No regime clássico, os feixes serão separados por cada analisador de polarização em partes iguais, e todos os detectores da figura 2 registrarão a mesma intensidade de luz.

O que acontece no regime quântico? Um pulso monofotônico ${ }^{4}$ que entra no interferômetro terá iguais probabilidades de ser medido em cada um dos detectores. Se o fóton for detectado em $D_{1}$, isso significa que ele foi detectado com polarização o ; nesse caso, por retrodição, podemos inferir que ele seguiu pelo caminho $A$, que está associado à polarização a $0^{\circ}$. Para confirmar isso, podemos bloquear o caminho $A$, e verificar que todos os fótons caem em $D_{3}$ e $D_{4}$. Assim, as trajetórias podem ser conhecidas, e o fenômeno é corpuscular. O princípio de complementaridade pode ser testado, girando lentamente o defasador $H$ que se encontra no caminho $A$, para verificar se ocorre alguma variação nas taxas de contagem dos detectores (o que corresponderia a um padrão temporal de interferência). Como tal variação não ocorre, o fenômeno claramente não é ondulatório (no sentido dado ao termo por Bohr).

De acordo com a interpretação da complementaridade, no caso em discussão pode-se representar o fóton detectado como uma partícula que seguiu um caminho bem determinado. Se a partícula passou pelo rotor $R$, então seu estado de polarização foi alterado; se não, então seu estado permaneceu o mesmo. Essa "informação" é carregada pela partícula ao longo de sua trajetória, até sua detecção.

A análise desse fenômeno corpuscular, segundo a interpretação da complementaridade, segue linhas diferentes da caracterização feita pela física ondulatória clássica. Para esta, a onda eletromagnética que se propaga entre $S_{2}$ e $P_{1}$ está de fato polarizada a $45^{\circ}$, e não a $0^{\circ}$ ou $90^{\circ}$ (ou seja, ela está, de fato, em uma superposição vetorial desses dois estados). É como se essa onda a $45^{\circ}$ não tivesse mais "memória” de quais eram as suas componentes originais. É possível estender tal interpretação para o domínio quântico, no que pode ser chamada "interpretação ondulatória realista" (Pessoa, 2003, p. 13).

4 Ou seja, um pulso que levará a um único disparo de um detector de eficiência perfeita. Poderíamos ter escrito “um fóton”, mas é preferível reservar o termo para um evento medido de detecção de luz. 
Refinamentos DA interpretação DA GomplementaRidade...

Enfim, trata-se de duas maneiras distintas de interpretar a situação. A adoção da retrodição não leva a inconsistências, desde que se mantenha no interior de uma mesma interpretação. Pode-se até fornecer interessantes "argumentos de plausibilidade" em favor da retrodição (cf. Vaidman et al., 1987), mas não os examinaremos aqui.

\section{Modificando o tipo de Fenômeno Sem ALteração do ESTAdo}

Considere-se agora um filtro de polarização $F$, que transmite totalmente o feixe de luz que está linearmente polarizado a $45^{\circ}$, e que bloqueia completamente um feixe de luz polarizado a $-45^{\circ}$. Suponhamos que $F$ seja inserido entre $S_{2}$ e $P_{1}$, conforme ilustrado na figura 2 (cf. Pessoa, 2003, p. 176). O que irá acontecer?

A orientação do polarizador é escolhida de forma a deixar inalterado o feixe de luz entre $S_{2}$ e $P_{1}$. Em outras palavras, o feixe está em um auto-estado do operador de projeção (de autovalor 1) associado ao filtro. O resultado dessa escolha é que o estado quântico do sistema não é alterado pela inserção do filtro $F$ e, assim, não ocorrem alterações nas probabilidades de medição em cada um dos detectores.

Porém, o tipo de fenômeno associado ao detector $D_{1}$, que anteriormente era corpuscular, agora torna-se ondulatório! Para mostrar que não é corpuscular, pode-se bloquear o caminho $A$, e verificar que mesmo assim incidem fótons em $D_{1}$. Para confirmar que é ondulatório, basta girar lentamente o defasador $H$ que se encontra no caminho $A$, e observar que uma variação na fase $\phi$ de fato leva a variações nas taxas de contagem em $D_{1}$ e $D_{3}$, proporcionais ao $\cos ^{2} \phi .5$ filtro que foi inserido age como um "apagador quântico" (cf. Herzog et al., 1995); pode-se dizer (no contexto da interpretação da complementaridade) que a informação de caminho foi destruída pelo filtro. Assim, os fótons detectados passam a ser associados a um fenômeno ondulatório, em que não há informação de caminho.

Note-se que a variação em $\phi$, mencionada acima, não altera as taxas de detecção em $D_{2}$ e $D_{4}$. Os fenômenos associados a esses detectores continuam corpusculares, enquanto que os associados a $D_{1}$ e $D_{3}$ são ondulatórios. Isso mostra que o conceito de "fenômeno" refere-se separadamente a cada quantum individual que é detectado.

Como pode a modificação de uma parte da aparelhagem, sem alterar o estado do objeto quântico, mudar o tipo de fenômeno? Isso não é tão surpreendente, se lembrar-

5 Quando a fase é mudada do valor inicial $\phi=$ o para um valor diferente de zero, parte do feixe de luz passa a ser absorvido pelo filtro, alterando, nesses casos, o estado do objeto quântico. Porém, permanece verdade que, para $\phi=$ o, não há alteração do estado quântico. A mudança em $\phi$ é um teste operacional para checar se o fenômeno associado a $\phi=$ o é ondulatório. A conclusão do teste é que, quando $\phi=0$, o fenômeno é de fato ondulatório (segundo a definição de Bohr). Se o teste for feito sem o filtro, não haverá absorções, e as taxas de contagem permanecerão as mesmas. 
mos da afirmação de Bohr (1995 [1949], p. 51) de que o fenômeno deve ser definido para a "totalidade" envolvendo objeto mais aparelhagem. Ora, se a aparelhagem é modificada, isso pode mudar a sua relação com o objeto (mesmo sem haver absorção), mudando, assim, o tipo de fenômeno. ${ }^{6}$ Isso exemplifica a ideia de que o conceito de fenômeno é relacional, envolvendo a relação entre objeto e aparelho (cf. Jammer, 1974, p. 166, 198).

Outra maneira de entender a situação é considerar que a caracterização de um fenômeno ondulatório, por meio de franjas de interferência (espaciais ou temporais), envolve uma coleção de arranjos experimentais levemente diferentes (diferentes valores da defasagem $\phi$ ). No presente estudo de caso, calhou de haver um valor de defasagem para o qual não ocorre absorção ou qualquer alteração do estado quântico. ${ }^{7}$ Enfim, caracterizar um fenômeno como ondulatório é dizer algo sobre uma classe de arranjos experimentais próximos entre si.

Ainda outra maneira de entender essa situação é considerar (como já vimos) que se o pacote monofotônico estivesse vindo por $A$, carregando a correspondente informação de polarização $\left(\mathrm{a} \circ^{\circ}\right)$, o filtro $F\left(\mathrm{a} 45^{\circ}\right)$ certamente alteraria o estado do pacote, ou absorvendo-o ou modificando seu estado de polarização. $O$ mesmo valeria para o componente vindo de $B$. Como tais componentes são implicitamente usados na definição do fenômeno corpuscular, segue-se que a inserção do filtro pode modificar o fenômeno, mesmo que o estado quântico (a soma dos componentes) não seja alterado.

\section{O EXPERIMENTO DE UNRUH}

Outro exemplo de "modificação do tipo de fenômeno sem alteração do estado quântico" foi descrito pelo físico canadense William Unruh, em seu argumento, publicado apenas na internet (cf. Unruh, 2004), de que o paradoxal experimento proposto por Afshar não é, de fato, uma refutação do princípio de complementaridade de Bohr.

\footnotetext{
6 Bohr chegou a essa conclusão em sua explicação do célebre problema levantado por Einstein, Podolsky \& Rosen, em 1935. Consideremos a montagem sugerida por David Bohm, envolvendo a medição de componentes de spin de pares de partículas correlacionadas (cf. Bohm 1951, p. 614). Imãs de Stern-Gerlach são usados para separar estados ortogonais das partículas, antes da detecção (diferentes orientações de um imã correspondem a diferentes observáveis sendo medidos). Nesse exemplo, a alteração de um imã, mesmo localizado a uma grande distância de uma das partículas, alteraria a totalidade do fenômeno, alterando assim "as próprias condições que definem os tipos possíveis de previsões relativas ao comportamento futuro do sistema” (Bohr, 1981 [1935], p. 103-4). Porém, ao contrário do que acontece no experimento examinado no texto, uma mudança na orientação de um imã altera o estado quântico do objeto que passa por ele.

${ }_{7}$ No interferômetro sem polarizadores (ver fig. 1), há valores de defasagem em $H(\phi=\pi / 2$ ou $3 \pi / 2$ ) que levam a taxas de contagens idênticas ao fenômeno corpuscular obtido pela remoção de $S_{2}$. Nesses casos, também não há absorção do feixe, mas os estados quânticos das duas situações são distintos (ao contrário da situação examinada no texto).
} 


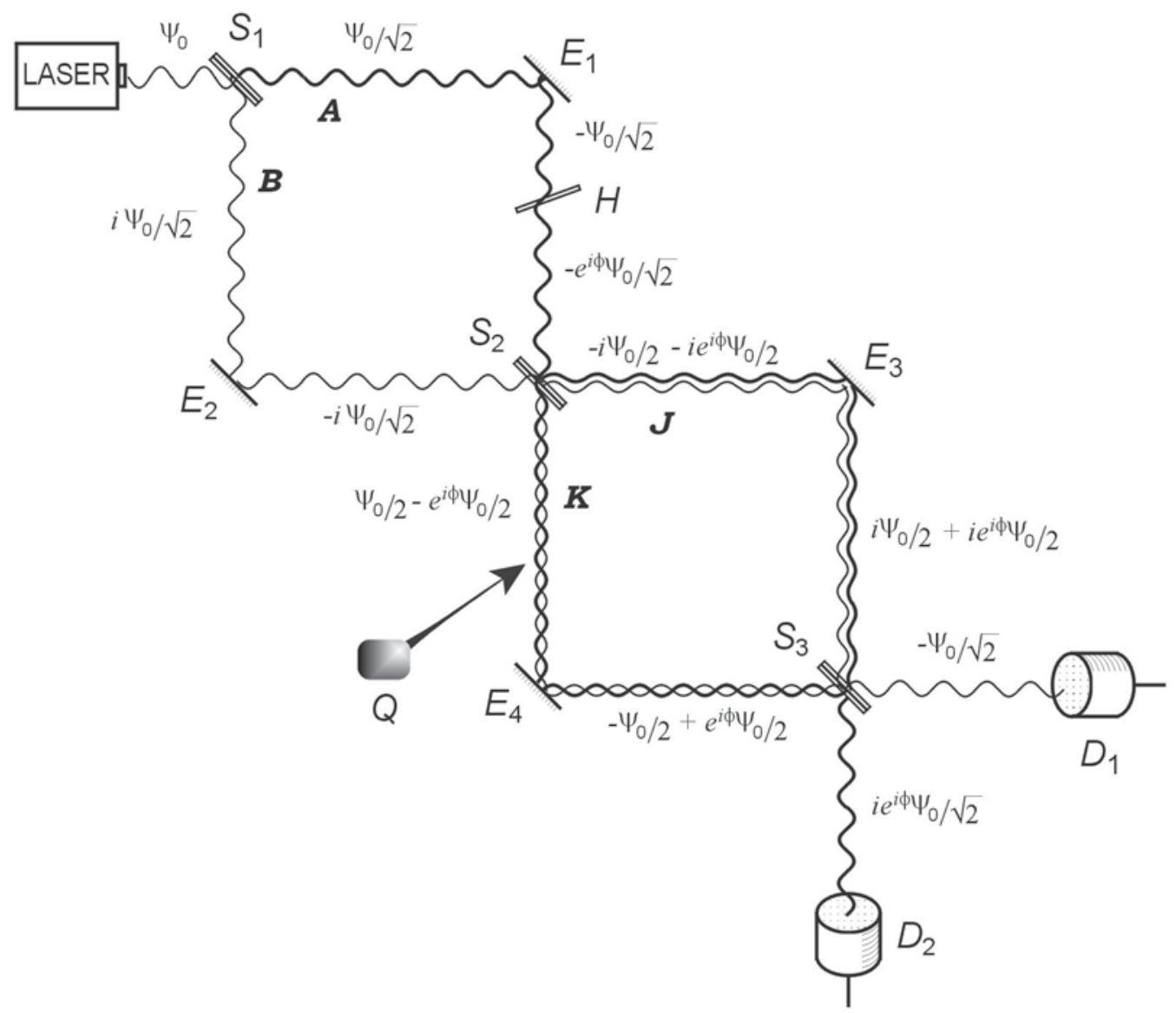

Figura 3. Experimento de Unruh. A montagem consiste de dois interferômetros de Mach-Zehnder em série. Nessa situação, os fótons detectados em $D_{2}$ correspondem a um fenômeno corpuscular, contendo informação de que vieram pela trajetória $A$, desenhada com linha mais escura. Se um bloqueador $Q$ for inserido no caminho $K$, nenhuma luz é absorvida, mas os fótons medidos nos detectores passam a estar associados a um fenômeno ondulatório no interferômetro $A B$.

A montagem de Unruh consiste de dois interferômetros de Mach-Zehnder em série, representados na figura. $3^{\mathbf{8}}$ Nessa situação, os fótons que são detectados em $D_{2}$ estão associados a um fenômeno corpuscular no primeiro interferômetro $A B$, correspondendo à trajetória $A$ (e os fótons de $D_{1}$ estão associados ao trecho $B$ ). Isso pode ser comprovado bloqueando-se o caminho $B$, e verificando que nenhuma luz chega em $D_{1}$ (e analogamente ao se bloquear o trecho $A$ ). A trajetória dos fótons de $D_{2}$ é indicada

$8 \mathrm{Na}$ figura 3, estão indicadas as amplitudes das ondas em cada trecho, adotando-se a seguinte regra. Em espelhos semirrefletores, a transmissão não modifica a fase da onda, mas a reflexão introduz um fator de fase $i$ (avanço de $1 / 4$ de onda). Nos espelhos de reflexão total, o fator de fase introduzido é -1 (avanço de meia onda). O defasador $H$ introduz uma fase arbitrária de $\phi$. 
na figura pelas linhas onduladas mais grossas, que se recombinariam em $S_{3}$ gerando interferência construtiva em direção a $D_{2}$, e destrutiva em direção a $D_{1}$. Porém, quando os dois componentes ( $A$ e $B$ ) se propagam, sem bloqueio, deixa de haver interferência construtiva em $S_{3}$, pois não há luz se propagando pelo caminho $K$. De qualquer maneira, não há dúvida de que o fenômeno associado à detecção em $D_{2}$ é corpuscular (no sentido da interpretação de Bohr) para todo trajeto do interferômetro (analisaremos isso com mais detalhe na seção 9). Outro teste é que, na montagem da figura 3, a rotação da lâmina $H$ (localizada no caminho $A$ ) não gera variação nas taxas de detecção nos dois medidores (como ocorreria em um fenômeno ondulatório).

O tipo de fenômeno pode ser modificado, sem alterar o estado quântico da luz, ao inserir um bloqueador no caminho $K$. Está claro que nenhum fóton será absorvido pelo bloqueador, pois neste caminho há interferência destrutiva da luz. Mesmo assim, verifica-se que agora os fótons detectados em $D_{1}$ e $D_{2}$ estão associados a um fenômeno ondulatório em $A B$ (e corpuscular em $J K$ ). Se a luz for impedida de seguir pelo caminho $B$, o feixe vindo de $A$ cairá nos dois detectores $D_{1}$ e $D_{2}$. Além disso, a lenta rotação da lâmina $H$ levará a um padrão temporal nítido de máximos e mínimos (igual em ambos os detectores), o que caracteriza um fenômeno ondulatório.

\section{O experimento de Afshar}

O experimento proposto por Shahriar Afshar, a partir de 2001, parte da montagem apresentada na figura 4 , que é o bem conhecido experimento da dupla fenda (cf. Pessoa, 2003, p. 3, 154). Luz coerente, como a de um laser, incide pela esquerda em duas fendas finas ( 1 e 2). A difração que ocorre em cada fenda é indicada pelas duas flechas, sendo que a luz se espalha como uma onda dentro dos limites representados pelas flechas. Nas regiões em que os feixes vindos de 1 e 2 se encontram, no anteparo à direita, forma-se um padrão de franjas de interferência, com regiões de claro e escuro.

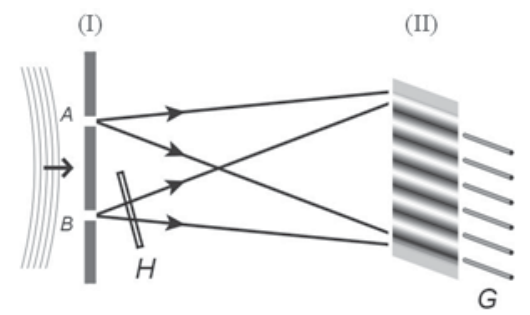

Figura 4. Experimento da dupla fenda. Este é o ponto de partida do experimento de Afshar, a tradicional montagem que gera franjas de interferência (com claros e escuros). Indica-se à direita o posicionamento da grade de fios metálicos $G$, que será inserida nas regiões escuras das franjas. A rotação da lâmina $H$ gera um deslocamento vertical das franjas. Veremos na seção 8 que o fenômeno associado à detecção de um fóton na região (II) é ondulatório com referência ao plano das fendas (I), mas corpuscular com relação ao próprio plano (II). 
Refinamentos da interpretação da Gomplementaridade...

$\mathrm{Na}$ figura 4, está desenhado à direita um conjunto de fios metálicos $G$ que, no experimento de Afshar, são inseridas justamente nas faixas escuras das franjas de interferência. Além disso, representa-se na figura uma lâmina de vidro $H$, que tem a mesma finalidade que aquela usada na figura 1 , ou seja, ao ser lentamente girada, a fase da luz que sai pela fenda 2 é continuamente alterada. No experimento da figura 4, essa variação de fase leva a um deslocamento contínuo das franjas, o que é típico de um fenômeno ondulatório.

O experimento montado por Afshar e sua equipe (cf. Afshar et al., 2007), mostrado na figura 5 , utiliza orifícios circulares (ao invés de fendas compridas) e faz uso de uma lente convexa fina $L$. No plano de imagem dessa lente, são colocados dois detectores $D_{1}$ e $D_{2}$, cada qual discernindo a luz que vem de uma das fendas. A situação é análoga ao que acontece em um microscópio simples, em que as imagens das fendas são focadas de maneira invertida no plano de imagem.

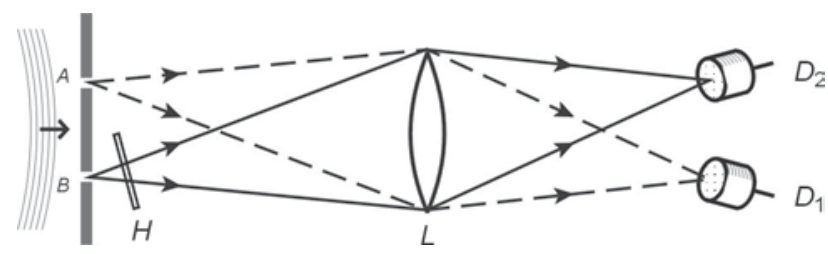

Figura 5. Experimento da dupla fenda com lente fina. $O$ uso da lente $L$ redireciona a luz de acordo com sua direção de propagação, e os detectores (colocados no plano de imagem da lente) são sensíveis à fenda por qual passou a luz: $D_{1}$ recebe apenas luz que passa pela fenda $A, \mathbf{e} D_{2}$ apenas da fenda $B$. A rotação de $H$ não gera alteração nas taxas de contagem, confirmando que o fenômeno é corpuscular com respeito às fendas (mas ondulatório com relação à região da lente).

Claramente, os fótons que são detectados em $D_{1}$ podem ser associados, por retrodição, à fenda $A$, e os fótons medidos em $D_{2}$ à fenda $B$. Se uma das fendas for bloqueada, o detector correspondente deixa de receber luz. Além disso, a variação de $H$ não leva a uma mudança nas intensidades medidas nos detectores. Isso confirma que, relativo ao plano das fendas, o fenômeno é corpuscular, ao contrário do que ocorre no experimento simples da dupla fenda (Fig. 4). O ponto em que cada fóton atravessa a lente não pode ser determinado, de forma que o fenômeno é ondulatório com respeito a esta parte do trajeto.

A novidade de Afshar foi introduzir a grade de finos fios metálicos $G$, colocados justamente nas regiões escuras das franjas de interferência. Antes de estudarmos a montagem final de Afshar, vale à pena ver se a grade não destrói, de maneira significativa, a informação acerca de a partir de qual fenda veio cada fóton detectado. No arran- 


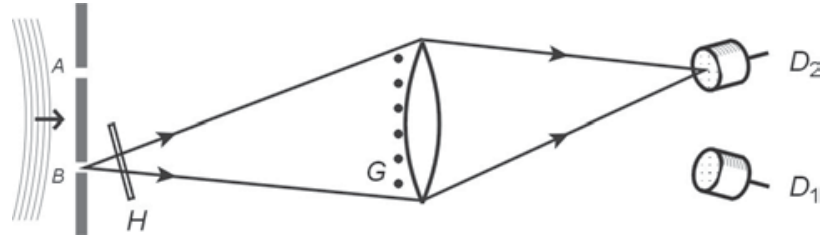

Figura 6. Experimento com inserção da grade $G$ à frente da lente fina. Com apenas uma fenda aberta (indicada na figura), $15 \%$ da luz é espalhada ou absorvida pela grade, mas a rotação de $H$ não gera alteração na taxa de contagem em $D_{2}$, confirmando que o fenômeno é corpuscular (com relação ao plano das fendas, mas não à região do prisma).

jo da figura 6, há apenas o feixe de luz que é difratado pela fenda $B$. Esse experimento foi feito por Afshar e colaboradores (Afshar et al., 2007, p. 299), que constataram que $15 \%$ da luz é bloqueada ou desviada pela grade (de forma que $85 \%$ dela chega a $D_{2}$ ). Parte dessa luz é difratada, resultando em um sinal de em cerca de $0,45 \%$ no detector $D_{1}$. No geral, isso corresponde a um fenômeno intermediário (relativo ao plano das fendas) muito próximo ao corpuscular, com proporção de 99\% corpuscular e $1 \%$ ondulatório. 9

O experimento final de Afshar envolve luz vinda de ambas as fendas, conforme a figura 7, com grade e lente. A influência da grade nas intensidades detectadas é bem menor do que no caso anterior, e as intensidades caem para 98\% do valor sem grade (Afshar et al., 2007, p. 299).

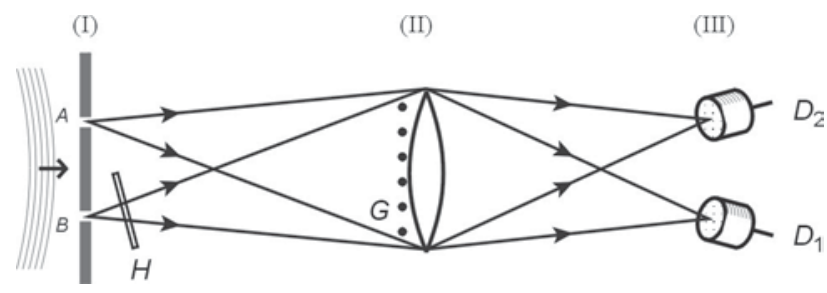

Figura 7. Experimento de Afshar. Com as duas fendas abertas, a grade não absorve praticamente nenhuma luz. Ao contrário da figura 4 , o fenômeno associado a um quantum detectado é corpuscular com referência à região (I) e ondulatório com referência à região (II). (Figura modificada a partir de Afshar et al., 2007.)

O físico iraniano interpretou a situação da seguinte maneira. Como a inserção da grade praticamente não atenua o feixe, isso indica que há regiões escuras defronte à lente, o que é uma característica ondulatória. Por outro lado, vimos na figura 6 que, quando uma única fenda é aberta, praticamente um único detector dispara, de forma que cada detector fornece informação sobre a trajetória no plano das fendas. É verdade que não há informação de trajetória na região da grade e lente, mas mesmo assim ob-

9 A "visibilidade" de um padrão de interferência define-se a partir dos pontos máximo e mínimo de intensidade: $V$ = $(I M A X-I \min ) /(I M A X+\operatorname{Imin})$. Em fenômenos nitidamente (100\%) ondulatórios, $V=1$, e em fenômenos nitidamente corpusculares, $V=0$. Os chamados "fenômenos intermediários" correspondem a estados puros que levam a uma visibilidade diferente de o e 1 (cf. Wootters \& Zurek, 1979). A visibilidade é uma medida direta da fração em que um fenômeno é ondulatório (por exemplo, um fenômeno $25 \%$ ondulatório gera um padrão de visibilidade $V=1 / 4$ etc.) 
Refinamentos da interpretação da Gomplementaridade...

tém-se um resultado surpreendente: constatam-se franjas de interferência juntamente com informação de trajetória. Segundo Afshar, essa conclusão violaria o princípio de complementaridade de Bohr.

\section{O TIPO DE FENÔMENO REFERE-SE A UM \\ TRECHO PERGORRIDO PELO OBJETO QUÂNTICO}

Antes de prosseguirmos a análise das conclusões de Afshar, é preciso salientar que o tipo de fenômeno, associado a um quantum detectado em algum medidor, pode variar conforme o trecho referido. Comecemos pelo interferômetro de Mach-Zehnder simples, da figura 1. O fenômeno é claramente ondulatório na região $A B$, entre os espelhos semirrefletores $S_{1}$ e $S_{2}$, mas é corpuscular na região antes de $S_{1}$ e naquela após $S_{2}$, na medida em que podemos determinar sem ambiguidades a trajetória nestes trechos. $\mathrm{Na}$ figura 3, também temos que deixar claro a qual trecho estamos nos referindo, se ao interferômetro $A B$, ao $J K$, ou ao trecho entre $S_{3}$ e o detector em questão.

Todo detector envolve uma medição direta da posição do quantum. Assim, para qualquer detector, é razoável afirmar que o fenômeno associado a um quantum detectado, em referência à região defronte ao próprio detector, é sempre corpuscular. Nos experimentos envolvendo o interferômetro de Mach-Zehnder, essa conclusão é trivial, mas, no experimento da fenda dupla, isso leva a uma constatação contra-intuitiva. Pois na figura 4, se colocarmos um detector na região de interferência (II), o fóton detectado estará associado a um fenômeno ondulatório em referência ao plano das fendas (I) (pois não há informação de trajetória nesse plano), mas a um fenômeno corpuscular em referência ao próprio plano de interferência (II).

Essa constatação serve para unificar o que Bohr chamou de "postulado quântico" com seu princípio de complementaridade (em outros termos, unifica a dualidade ondapartícula "fraca" com a "forte" (cf. Pessoa, 2003, p. 3-4, 17-8). O quantum detectado em uma tela fosforescente aparece sempre na forma de um ponto. Ora, isso sempre corresponde a um fenômeno corpuscular, mas apenas com referência à região adjacente à tela. Afinal, sabemos sem ambiguidades a posição do quantum nessa região. Porém, se o fenômeno é corpuscular ou ondulatório com referência a uma região mais distante, isso dependerá da montagem experimental.

Aplicando essas ideias ao experimento de Afhsar (Fig. 7), inicialmente sem a grade $G$, constatamos imediatamente que os fótons detectados em (III) correspondem a um fenômeno corpuscular com relação ao plano das fendas (I), e a um fenômeno ondulatório com relação ao plano (II) diante do prisma. Veremos, na seção seguinte, uma situação análoga com o interferômetro de Unruh. 
A inserção da grade modifica a situação apenas na medida em que transforma um fenômeno $100 \%$ corpuscular (com relação ao plano das fendas) em um fenômeno $99 \%$ corpuscular e $1 \%$ ondulatório. Este pequeno componente ondulatório pode ser interpretado como uma modificação do tipo de fenômeno sem alteração do estado quântico, como fez Unruh (2004). (Voltaremos a esse ponto na seção 10.)

\section{VARIAÇÃO DAS FASES NO EXPERIMENTO DE UNRUH}

Vale a pena examinarmos com mais cuidado o experimento de Mach-Zehnder duplo de Unruh (seção 6), explorando a analogia entre essa montagem e o experimento de Afshar. Consideraremos agora a inserção de dois defasadores, $H_{1}$ e $H_{2}$, cada qual em um dos interferômetros da montagem de Unruh, conforme indicado na figura 8.

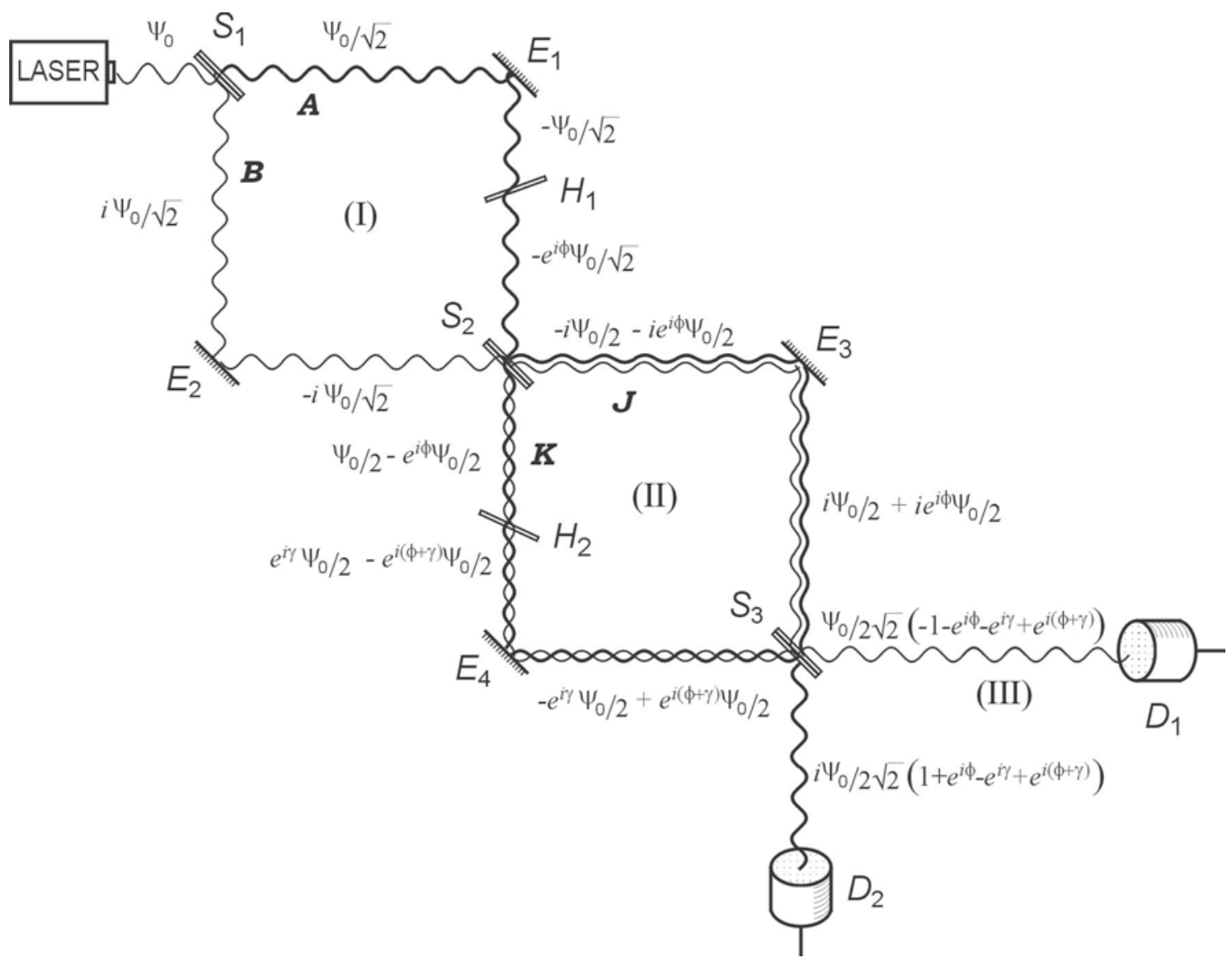

Figura 8. Experimento de Unruh com dois defasadores. As amplitudes de ondas estão indicadas para cada trecho, onde as fases introduzidas pelos defasadores $H_{1} \mathbf{e} H_{2}$ são respectivamente $\phi \mathbf{e} \gamma$. 
Refinamentos da interpretação da Gomplementaridade...

Vemos, na figura, que a amplitude que chega ao detector $D_{1}$ é

$$
\psi_{1}=\frac{\psi_{0}}{2 \sqrt{ } 2}\left(-1-e^{i \phi}-e^{i \gamma}+e^{i(\phi+\gamma)}\right),
$$

onde $\psi_{0}$ é a amplitude inicial, e $\phi$ e $\gamma$ são as fases introduzidas em $H_{1}$ e $H_{2}$. Usando a regra de Born para a intensidade (probabilidade) em $D_{1}, I_{1}=\left|\psi_{1}\right|^{2}$, obtemos:

$$
I_{1}=\frac{\left|\psi_{0}\right|^{2}}{2}(1+\operatorname{sen} \phi \cdot \operatorname{sen} \gamma)
$$

Essa expressão indica uma simetria com relação aos efeitos das fases $\mathrm{f}$ e g, onde a primeira refere-se ao interferômetro $A B$ (região I), e a segunda ao $J K$ (região II). Lembremos que se a variação da fase levar a uma oscilação na taxa de detecção, com visibilidade 1, então o fenômeno associado será nitidamente ondulatório. Examinaremos três casos:

CAso (1). No caso do experimento de Unruh (Fig. 3), $\phi=\gamma=0$. O fenômeno associado a uma detecção em $D_{1}$ é, portanto, corpuscular, tanto com respeito a $A B$ quanto a $J K$. Pois se variarmos $\phi$, não haverá alteração em $I_{1}$, indicando que o fenômeno é corpuscular para $A B$ (o fóton detectado será associado ao caminho $B$ ). Analogamente, mantendo $\phi=0$, se variarmos $\gamma$, não haverá mudança em $I_{1}$, indicando que o fenômeno é corpuscular também com referência a $J K$ (estando associado ao caminho $J$, o que fica claro pelo fato do feixe ser cancelado por $K$ ).

Caso (2). Suponha-se agora que a fase de $H_{1}$ seja fixada em $\phi=\pi / 2$, mantendo-se $\gamma=0$. Nesse caso, o fenômeno associado a $D_{1}$ continuará corpuscular com relação a $A B$ (pois a variação em $\mathrm{f}$ não altera $I_{1}$ ), mas será ondulatório com relação a $J K$. Na figura 8 , não teremos mais o cancelamento pelo caminho $K$, e a variação em g gera uma oscilação entre o e 1 em $I_{1}$. É este o caso que mais se aproxima da montagem de Afshar (Fig. 7), onde os tipos de fenômeno, nas regiões (I), (II) e (III) são, respectivamente, corpuscular, ondulatório e corpuscular.

CAso (3). A situação mais contra-intuitiva ocorre quando fixamos $\gamma=\pi / 2$, mantendo $\phi=0$. Ao fazermos isso, tornamos o fenômeno associado ao interferômetro $A B$ ondulatório, mesmo que o estado quântico do sistema não seja alterado. Trata-se de outro exemplo de "modificação do tipo de fenômeno sem alteração do estado quântico", só que, ao invés de inserir um bloqueador, como sugerido por Unruh (seção 6), insere-se uma lâmina de vidro que introduziria uma defasagem de $\pi / 2$, se houvesse um 
feixe passando por ela (mas não há!). Nesse caso, então, o fenômeno referente ao interferômetro $A B$ torna-se ondulatório, e o referente a $J K$ permanece corpuscular. Os fótons detectados tanto em $D_{1}$ quanto $D_{2}$ seguem pelo caminho $J$ (pois há interferência destrutiva por $K$ ).

Notamos que, apesar de a equação (1) ser simétrica em $\phi$ e $\gamma$, a interpretação da complementaridade introduz uma assimetria entre os casos (2) e (3), pois no caso (2) cada detector, $D_{1}$ e $D_{2}$, está associado a um caminho diferente, $B$ e $A$ respectivamente, ao passo que, no caso (3), cada detector está associado ao mesmo caminho J. Isso reflete a maior proximidade dos detectores ao interferômetro $K J$.

Notamos também que a situação característica de modificação do tipo de fenômeno sem alteração de estado é o caso (3), ao passo que a analogia mais próxima ao experimento de Afshar é o caso (2). Isso indica que o argumento de Unruh (2004) é insuficiente, pois ele se aplica a uma situação em que a região (II) está associada a um fenômeno corpuscular, passando do caso (1) para o caso (3), ao passo que, na montagem de Afshar, a região (II) está associada a um fenômeno ondulatório.

\section{Analogia interferométrica do experimento de Afshar}

Proporemos agora uma variação do experimento com interferômetros, para encontrar uma analogia mais adequada com o experimento de Afshar.

Considere-se a figura 9, construída de forma que o fenômeno associado ao fóton detectado em $D_{1}$, referente aos trechos $A B$, seja corpuscular, associado ao caminho $A$. Claro está que, referente ao trecho $L J K$, o fenômeno associado é ondulatório. Pois bem, a inserção do bloqueador $Q$, no caminho $K$, faz que metade da amplitude incidente em $D_{1}$ passe a estar associada a um fenômeno ondulatório, com referência ao trecho $A B$, como foi visto na figura 3. Assim, a inserção do bloqueador, mesmo sem absorver luz, gera, para os fótons detectados em $D_{1}$, um fenômeno intermediário com referência ao trecho $A B$, sendo 50\% ondulatório e 50\% corpuscular. Se as taxas de transmissão dos semi-espelhos $S_{4}$ e $S_{5}$ subissem para o,91, a inserção do bloqueador em $K$ geraria um fenômeno intermediário 99\% corpuscular, semelhante ao que ocorre no experimento de Afshar.

Nesse caso, então, podemos aplicar a análise de Unruh. A inserção da grade $\mathrm{G}$ na figura ๆ é análoga à inserção do bloqueador Q na figura 9. A diferença é que nesta última a absorção é rigorosamente zero, ao passo que no experimento de Afshar ocorre uma pequena absorção, devido à espessura finita dos fios da grade (e do fato de que o padrão só se anula, rigorosamente, em pontos isolados). Por outro lado, quanto mais 


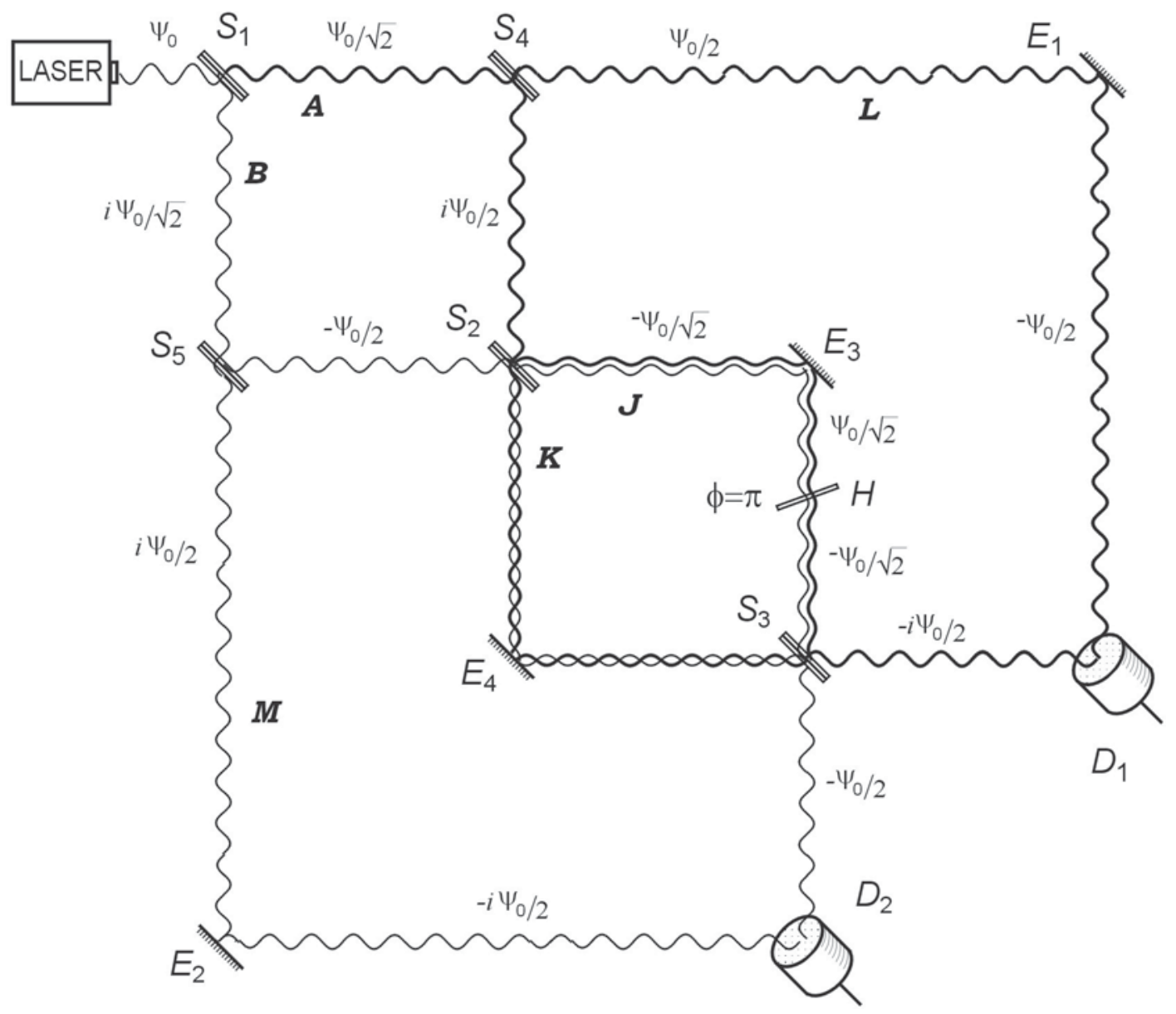

Figura 9. Fenômeno intermediário, em analogia com o experimento de Afshar. Neste arranjo interferométrico, sem a presença de um bloqueador no trajeto $K$, todos os fótons detectados em $D_{1}$ estão associados ao caminho $A$, estando assim associados a um fenômeno corpuscular referente à parte $A B$ (analogamente para $D_{2}$ ). $\mathrm{O}$ defasador $\mathrm{H}_{2}$ introduz uma fase fixa de $\pi$ (meio comprimento de onda). A inserção de um bloqueador $Q$ ( ou de um defasador fixado em $\pi / 2$, como vimos no caso 3 da seção 9 ) no caminho $K$ torna intermediário o fenômeno associado aos fótons detectados em $D_{1}$, que passa a ser $5 \circ \%$ ondulatório e $5 \circ \%$ corpuscular. Isso é confirmado girando lentamente o defasador $H_{1}$, o que leva a um padrão temporal de visibilidade $1 / 2 \mathrm{em} D_{1}$.

finos forem os fios da grade, mais corpuscular será o fenômeno associado aos fótons detectados (e referência ao plano das fendas).

Podemos concluir que o problema apontado por Unruh, de que a inserção da grade leva a uma mudança no tipo de fenômeno, mesmo sem alterar o estado quântico, de fato ocorre, mas em uma proporção que pode ser desprezada no experimento de Afshar. 


\section{Conclusão}

A análise precedente sublinhou um ponto simples mas importante com relação ao experimento de Afshar. Ao formularmos a questão de qual é o fenômeno associado a um fóton medido em um dos detectores da figura 7, devemos deixar claro a qual trecho estamos fazendo referência. Se for ao trecho (I), próximo das fendas, o fenômeno será corpuscular; se for ao trecho (II), defronte à lente, ele será ondulatório (e, claro, referente ao trecho (III), próximo aos detectores, ele é sempre corpuscular).

Notamos também que se o detector for colocado na própria região de interferência, como a grade $G$ na figura 4, o fenômeno referente ao plano das fendas será ondulatório. E, curiosamente, o fenômeno associado à própria região (II) (encostada em cada detector) será corpuscular.

De qualquer forma, o experimento de Afshar não traz problemas para a interpretação da complementaridade, no que se refere à identificação de qual o tipo de fenômeno associado aos detectores $D_{1}$ e $D_{2}$ da figura 7 . Basta adotarmos o refinamento conceitual de que o tipo de fenômeno refere-se a trechos do experimento.

A grande novidade do experimento de Afshar é mostrar que é possível "constatar" simultaneamente o aspecto corpuscular associado à região (I) e o aspecto ondulatório associado à região (II). Para tornar essa afirmação mais precisa, pode-se substituir os fios metálicos da grade $G$ por fios detectores, capazes de medir a incidência de luz. Nesse caso, a medição nula de luz (ou quase nula, devido à espessura finita de cada fio) é uma indicação indireta de que o fenômeno detectado em $D_{1}$ e $D_{2}$, referente à região (II), é ondulatório.

Consideremos o experimento da figura 9, com taxas de transmissão dos semiespelhos $S_{4}$ e $S_{5}$ fixados inicialmente em o,5. Fica claro que a inserção de um detector em $K$ não altera o tipo de fenômeno na própria região (II), que inclui as trajetórias $J$ e $L$, que permanece $100 \%$ ondulatório. Quando as taxas de transmissão dos semi-espelhos $S_{4}$ e $S_{5}$ são alteradas para o,91, o fenômeno associado às trajetórias J e $L$ passa a ser $99 \%$ corpuscular, mas a inserção do detector ou bloqueador em $K$ não altera o tipo de fenômeno na própria região (II). No entanto, a inserção altera o tipo de fenômeno na região (I), que passa de 100\% corpuscular para 50\% corpuscular (no caso da taxa de transmissão de 0,5 ) ou para $99 \%$ corpuscular (no caso da taxa de o,91).

Avaliemos agora o impacto do experimento de Afshar sobre a interpretação da complementaridade. Levando em conta que a atribuição do tipo de fenômeno deve fazer referência ao trecho do experimento, o experimento de Afshar não traz nenhum problema para essa atribuição do tipo de fenômeno. $\mathrm{O}$ experimento tem o mérito de mostrar a importância de levar em consideração o trecho envolvido, mas esse esclarecimento não mina a coerência da interpretação de Bohr. Um esclarecimento do prin- 
cípio de complementaridade que teve efeitos semelhantes ocorreu na descoberta de que existem fenômenos intermediários (cf. Wooters \& Zurek, 1979).

Resta, porém, analisarmos a questão da determinação simultânea de fenômenos em dois trechos diferentes (para um mesmo quantum detectado em um detector específico). O mérito de Afshar é ter mostrado que isso pode ser feito de maneira satisfatória.

Em primeiro lugar, há a determinação da trajetória do objeto quântico na região (I) das fendas. Essa determinação é indireta, e obtida a partir de experimentos em que apenas uma das fendas está aberta (como na figura 6, sem grade), resultando em apenas um detector disparando, ou por meio da variação contínua da fase de um componente logo após a saída da fenda (como na figura 5 ). Esses critérios são aceitos pela interpretação da complementaridade. ${ }^{10}$

Em segundo lugar, há a constatação das regiões escuras do padrão de interferência ondulatório, obtido pela inserção da grade $G$, como indicado na figura 4. A novidade do experimento de Afshar é argumentar que tal procedimento, que pode ser considerado uma medição de resultado nulo (especialmente se os fios da grade forem substituídos por fios medidores), de equivale, de fato, a uma determinação do caráter ondulatório do fenômeno, referente ao trecho (II). Parece-nos que tal conclusão é correta.

O que o clássico debate entre Bohr e Einstein (cf. Bohr 1995 [1949]) havia mostrado é que um quantum detectado na própria região de interferência, a saber, a região (II), não pode dar indício nítido de trajetória na região (I) das fendas. O experimento de Afshar não viola esse resultado, pois se a grade $G$ for introduzida como um medidor, qualquer fóton detectado por ela não poderá ser associado a uma trajetória vinda de uma fenda.

Por outro lado, há pouquíssimos fótons absorvidos na grade, e esse número tende a zero à medida que a espessura da grade tendesse a zero. Portanto, quase nenhum fóton que chega em $D_{1}$ e $D_{2}$ "teve apagada sua informação de trajetória" (para usar uma expressão imprecisa), ou melhor, o fenômeno associado aos detectores (e referente à região I) é intermediário em uma proporção muito pequena do caráter ondulatório (estimamos 1\% com base nos dados de Afshar).

Em suma, não há porque considerar o experimento uma violação do princípio de complementaridade, mas sim uma extensão ou um refinamento do princípio. O trabalho de Afhsar não é nem revolucionário, nem trivial. Ele mostrou algo que ninguém tinha mostrado, mas que pode ser acomodado dentro da interpretação da complemen-

10 Kastner (2005, p. 656-7) critica o argumento de Afshar, negando que se possa asseverar qual é a fenda pela qual o fóton "de fato atravessou". Mas ao argumentar desta maneira, ela está fugindo da interpretação da complementaridade, e aproximando-se talvez de uma visão mais realista ondulatória, para a qual a luz passa simultaneamente por ambas as fendas, qualquer que seja o observável posteriormente medido. Além do mais, a interpretação da complementaridade concorda que não se pode fazer afirmações "de fato” para eventos não observáveis. 
taridade. Para isso, o essencial é salientar que, em um mesmo experimento, há regiões em que o objeto quântico detectado pode ser associado a fenômenos diferentes, ora sendo associado a um fenômeno corpuscular (nas fendas), ora a um ondulatório (diante do prisma). É isso que está expresso, de maneira mais realista, na epígrafe bohriana de Bohm, que abre este artigo.@

\author{
Osvaldo Pessoa Júnior \\ Professor do Departamento de Filosofia, \\ Faculdade de Filosofia, Letras e Ciências Humanas, \\ Universidade de São Paulo, Brasil. \\ opessoa@usp.br
}

\title{
Refinements of the interpretation of complementarity from Afshar's experiment
}

\begin{abstract}
Afshar's experiment was recently proposed as a violation of the principle of complementarity. Acknowledging the novelty brought by the experiment, we argue that it allows a refinement of this principle, with the establishment of two points: (1) the possibility of modifying the "type" of phenomenon (wave or particle) without changing the quantum-mechanical state, and (2) the recognition that the type of phenomenon, associated to a detected quantum, refers to a specific region traversed by the quantum object. The first point is explored in Mach-Zehnder interferometers, with polarization devices and in Unruh's double setup. The second point emphasizes that a phenomenon can be corpuscular with respect one region and undulatory with respect to another. Afshar's originality lies in his proposal of a way of ascertaining both simultaneously. Restriction (1) turns out having a negligible effect in Afshar's experiment.
\end{abstract}

KEYwORDs $\bullet$ Philosophy of physics. Foundations of quantum mechanics. Complementarity. Wave-particle duality. Quantum-mechanical state. Mach-Zehnder interferometer. Retrodiction. Afshar's experiment. Unruh's experiment.

\section{REFERÊNGIAS BIBLIOGRÁFICAS}

Afshar, S. S. Sharp complementary wave and particle behaviors in the same welcher weg experiment. 2003. Disponível em: 〈http://www.irims.org/quant-ph/o3०5०3〉. Acesso em: 28 fev. 2012.

Afshar, S. S. et al. Paradox in wave-particle duality. Foundations of Physics, 37, p. 295-305, 2007.

Вонм, D. Quantum theory. Englewood Cliffs: Prentice-Hall, $195^{1}$.

Bонr, N. O postulado quântico e o recente desenvolvimento da teoria atômica. In: PessoA Jr., O. (Ed.). Fundamentos da física 1-Simpósio David Bohm. São Paulo: Livraria da Física, 2000 [1928]. p. 135-59. . A descrição da realidade física fornecida pela mecânica quântica pode ser considerada completa? Tradução G. W. Abramo. Cadernos de História e Filosofia da Ciência, 2, p. 97-106, 1981 [1935]. 
Refinamentos da interpretação da Gomplementaridade...

Вонг, N. O debate com Einstein sobre problemas epistemológicos na física atômica. In: .Física atômica e conhecimento humano, ensaios 1932-1957. Tradução V. Ribeiro. Rio de Janeiro: Contraponto, 1995 [1949]. p. 41-83.

Grangier, P. et al. Experimental evidence for a photon anticorrelation effect on a beam splitter: a new light on single-photon interferences. Europhysics Letters, 1, p. 173-9, 1986.

Heisenberg, W. The physical principles of the quantum theory. Chicago: University of Chicago Press, 1930. Hellmuth, T. et al. Delayed-choice experiments in quantum interference. Physical ReviewA, 35, p. 253241,1997 .

Herzog, T. J. et al. Complementarity and the quantum eraser. Physical Review Letters, 75, p. 3०34,-7, 1995 .

Kastner, R. E. Why the Afshar experiment does not refute complementarity. Studies in History and Philosophy of Modern Physics, 36, p. 649-58, 2005 .

Jammer, M. The philosophy of quantum mechanics. New York: Wiley, 1974.

Marlow, A. R. (Ed.), Mathematical foundations of quantum theory. New York: Academic, 1978.

Pessoa Jr., O. Complementing the principle of complementarity. Physics Essays, 13, p. 50-67, 2000 a. . (Ed.). Fundamentos da física 1 -Simpósio David Bohm. São Paulo: Livraria da Física, 200ob. . Conceitos da física quântica. São Paulo: Livraria da Física, 2003. v. 1.

UnRUH, W. Shahriar Afshar-quantum rebel? 2004. Disponível em: <http://axion.physics.ubc.ca/rebel. html>. Acesso em: 28 fev. 2012.

VaIdman, L. et al. How to ascertain the values of $\mathrm{s}_{\mathrm{x}}, \mathrm{s}_{\mathrm{y}}$, and $\mathrm{s}_{\mathrm{z}}$ of a spin-1/2 particle. Physical Review Letters,

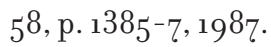

Wheeler, J.A. The "past" and the "delayed-choice" double-slit experiment. In: MarLow, A. R. (Ed.), Mathematical foundations of quantum theory. New York: Academic, 1978. p. 9-4,8.

Wootters, W. K. \& Zurek, W.H. Complementarity in the double slit experiment: quantum non-separability and a quantitative statement of Bohr's principle. Physical Review D, 19, p. 473-84, 1979. 